\section{A utilização de serviços odontológicos entre crianças e fatores associados em Sobral, Ceará, Brasil}

\author{
Use of dental care by children and associated \\ factors in Sobral, Ceará State, Brazil
}

\footnotetext{
1 Curso de Odontologia, Universidade de Fortaleza, Fortaleza, Brasil.

2 Programa de Pós-graduação em Ciências da Saúde, Universidade Federal do Rio Grande do Norte, Natal. Brasil.

${ }^{3}$ Curso de Odontologia Universidade Federal do Rio Grande do Norte, Natal, Brasil.

4 Secretaria de Estado do Ceará, Fortaleza, Brasil.

Correspondência L. R. A. Noro

Curso de Odontologia Universidade de Fortaleza. Av. Engenheiro Leal Lima Verde 2388, Fortaleza, CE 60833-520, Brasil. noro@unifor.br
}

\begin{abstract}
The aim of this study was to analyze the use of dental services by children in Sobral, Ceará State, Brazil, relating such use to socioeconomic factors and patterns of service consumption. An analytical cross-sectional study was performed, interviewing parents or guardians of 3,425 children. The relationship between independent variables and use of dental services was analyzed using tests of association (chi-squared), odds ratios $(\mathrm{OR})$ and respective confidence intervals, and multiple logistic regression. 50.9\% of children had used dental services at least once in their lives. Of these, $65.3 \%$ had used such services in the previous year and $85.4 \%$ in public dental services. The importance of the Brazilian Unified National Health System is highlighted by the response in terms of access to dental services, considering that the vast majority of treatment was provided by this public system. The factors that most affected use of dental services were socioeconomic status, health plan coverage, owning a toothbrush, regular garbage collection, maternal knowledge, sewage treatment, and malnutrition.
\end{abstract}

Health Services Accessibility; Dental Care for Children; Oral Health
Luiz Roberto Augusto Noro 1,2 Angelo Giuseppe Roncalli 3 Francisco Ivan Rodrigues Mendes Júnior 4 Kenio Costa Lima 2

\section{Introdução}

A Lei Orgânica da Saúde 1 preconiza como um de seus princípios o acesso universal e gratuito aos serviços e ações de saúde. A utilização dos serviços odontológicos é um item que permite uma aproximação a esse elemento, contribuindo para identificar como esse acesso pode ser obtido de forma a permitir a melhoria de saúde bucal da população brasileira.

A saúde bucal, na maioria dos municípios brasileiros, segundo Gomes et al. 2, constitui ainda um grande desafio aos princípios do Sistema Único de Saúde (SUS), principalmente no que se refere à universalização e à eqüidade do atendimento. Dados da Pesquisa Nacional por Amostra de Domicílios de 1998 (PNAD/1998) 3 demonstram que o atendimento odontológico se diferencia fortemente do atendimento médico, com uma proporção muito mais baixa de atendimentos financiados pelo SUS ou por planos de saúde, em comparação com os financiados diretamente. Tal situação remete à observada na Austrália 4 onde a cobertura universal de cuidados de saúde exclui o tratamento odontológico, fazendo com que de $80 \%$ a $90 \%$ dos procedimentos sejam custeados diretamente pelo consumidor ao sistema privado de assistência odontológica.

Apesar da reconhecida importância da saúde bucal, uma parcela considerável da população brasileira não tem acesso aos serviços de saúde 5 , dificultado pela falta evidente de articulação da 
luta pela sociedade brasileira por direitos relativos à saúde bucal ${ }^{6}$. Para Sanders \& Slade 7 , a percepção da população sobre a importância da saúde bucal está diretamente relacionada ao acesso ao serviço odontológico.

$\mathrm{O}$ aumento no emprego de medidas preventivas como a fluoretação das águas de abastecimento público, ações coletivas em saúde bucal, consumo de escova de dente, uso de dentifrício e educação em saúde, essenciais para melhoria das condições de saúde bucal, não permite, entretanto, que se perca de vista a importância da assistência odontológica em mitigar o sofrimento e buscar a recuperação de condições de saúde bucal, considerando-se, segundo Fernandes \& Peres 8, que o acesso aos serviços odontológicos no Brasil é limitado e desigual.

A própria Política Nacional de Saúde Bucal 9 atualmente em vigor reconhece a importância das ações de recuperação da saúde centradas no diagnóstico realizado o mais precocemente possível, assim como o tratamento instituído de imediato, de modo a deter a progressão da doença e impedir o surgimento de eventuais incapacidades e danos decorrentes, além de inverter a lógica que leva à mutilação, ainda hoje predominante em algumas situações no serviço público.

O objetivo deste estudo foi analisar a utilização de serviços odontológicos pela população infantil, na faixa etária de cinco a nove anos no Município de Sobral, Ceará, Brasil, relacionandoa com fatores sócio-econômicos e uso de serviços de saúde.

\section{Método}

A presente pesquisa caracteriza-se como estudo analítico transversal no qual foram entrevistados responsáveis por crianças na faixa etária de cinco a nove anos de idade, residentes na área urbana do Município de Sobral, nos anos de 1999 a 2000 .

A amostra foi composta por sorteio, com base no cadastro de domicílios elaborado por profissionais de saúde da Secretaria de Desenvolvimento Social e Saúde de Sobral, que incluía as crianças residentes na área urbana nascidas entre 1990 e 1994, estratificadas pelo ano de nascimento.

Para o cálculo da amostra foi considerada uma população estimada de 18.668 crianças na faixa etária de 5 a 9 anos de idade 10 . Tomando-se como referência uma prevalência de $10 \%$ entre as diversas situações previstas no questionário 11 e considerando uma margem de erro de $10 \% \mathrm{e}$ um nível de 95\% de confiança, o número final encontrado, ajustado para populações finitas, foi de
2.900 crianças. Entretanto, considerando-se a capacidade operacional disponível para a pesquisa, foram inicialmente sorteadas 4.400 (um acréscimo de cerca de $50 \%$ ), uma vez que pela característica da pesquisa era necessário prever as perdas (domicílio inexistente, idade errada no cadastro base, mudança de residência, viagem, negativa da família ou morte da criança). No total, foram realizadas ao longo do estudo 3.700 entrevistas domiciliares. Destas, 275 foram excluídas por inclusão inadequada de mais de uma criança no mesmo domicílio, duplicidade de questionário e preenchimento incompleto de entrevista domiciliar, ficando a amostra final composta por 3.425 crianças. Esse tamanho, que se revelou superior ao inicialmente calculado, permite estimar uma prevalência de $10 \%$ com margem de erro de $9 \%$ e mesmo nível de confiança.

O instrumento de trabalho foi um questionário semi-estruturado, aplicado por estudantes universitários de outras áreas que não a da saúde, especialmente capacitados para o desenvolvimento das entrevistas domiciliares, sob a coordenação de supervisores de campo. Como parte componente da capacitação foi realizada a calibração dos entrevistadores, para assegurar uma interpretação, compreensão e aplicação uniforme quanto aos padrões para as respostas registradas.

O estudo foi realizado por meio de entrevistas domiciliares, preferencialmente com a mãe da criança. Considerando-se a alta cobertura da Estratégia Saúde da Família na área urbana do $\mathrm{Mu}$ nicípio de Sobral (91,6\%), a grande maioria das entrevistas era agendada preliminarmente pelo agente comunitário de saúde responsável pela família, visando à maior facilidade na interação do pesquisador com a comunidade.

Para a elaboração do questionário 11 foram envolvidos vários pesquisadores de diferentes formações profissionais como médicos, cirurgiões-dentistas, enfermeiros, sanitaristas, estatísticos, entre outros. O instrumento foi composto por dez dimensões com seus respectivos itens: (a) identificação (dados gerais da criança); (b) características demográficas (sexo, idade, cor, religião, característica do domicílio, grau de parentesco com os moradores em sua residência e seus devidos graus de escolaridade, ocupação e renda mensal); (c) características da migração (local de nascimento e onde morou); (d) condições de moradia (tipo e localização do domicílio, característica da construção, abastecimento de água, esgoto e coleta de lixo, instalações sanitárias, posse de eletrodomésticos, proximidade e freqüência a atividades e serviços); (e) escolaridade da criança (freqüência à creche, pré-escola ou escola, repetência escolar, condição do 
aprendizado, faltas); (f) trabalho infantil (tipo, característica e motivo do trabalho, remuneração, horário, acidente de trabalho); (g) nutrição (desnutrição, participação em programa de desnutridos, merenda escolar); (h) morbidade referida nos últimos 15 dias (identificação do problema de saúde, atendimento, exame, solução do problema, gasto com a solução do problema, encaminhamento, internação hospitalar, medicação, atendimento odontológico); (i) situação vacinal (informação na carteira de vacinação, tipo de vacina, número de doses); e (j) consumo de serviços de saúde (vínculo com a Estratégia Saúde da Família, acesso, acolhimento, comunicação e relação com o serviço de saúde). No total, 266 itens eram perguntados, levando um tempo médio de quarenta minutos cada entrevista.

O desfecho analisado no presente estudo foi a utilização de serviços odontológicos pela população infantil de 5 a 9 anos de idade, por meio da questão objetiva: "A criança já foi alguma vez na vida ao dentista?", dicotomizada em "sim" ou "não".

Procurando identificar o papel das variáveis independentes existentes no estudo, após análise preliminar, os itens presentes nas dez dimensões foram agrupados em duas categorias: condição sócio-econômica e consumo de serviços. A Tabela 1 apresenta a distribuição das variáveis nessas duas categorias.

Os dados coletados foram duplamente digitados e processados usando-se o programa Epi Info versão 6.04 (Centers for Disease Control and Prevention, Atlanta, Estados Unidos). A va- lidação dos bancos foi realizada utilizando-se o recurso "VALIDATE" do Epi Info e, após limpeza dos dados, construído o banco de dados para análise final. A confiabilidade das informações coletadas pôde ser avaliada pela consistência do perfil das respostas comparadas aos resultados preliminares do estudo, assim como quando comparadas às características gerais da população do município.

Num primeiro momento, foram realizadas freqüências simples das variáveis independentes presentes no estudo e definidas nas situações "adequada" e "não adequada" para posterior avaliação por meio da análise univariada. Para avaliar a relação entre as variáveis independentes e a utilização de serviços odontológicos realizouse teste de associação pelo qui-quadrado, assim como se estimou a razão de chances (OR) e seu respectivo intervalo de confiança. As variáveis independentes com nível de significância $\mathrm{p}<0,20$ entraram na análise de regressão logística múltipla. Para definição do modelo de análise final, iniciou-se a regressão logística com a variável independente com maior significância estatística na análise univariada, adicionando-se as demais em ordem crescente a partir do procedimento "ENTER" do programa SPSS for Windows 13.0 (SPSS Inc., Chicago, Estados Unidos).

O trabalho foi previamente submetido e aprovado pelo Comitê de Ética em Pesquisa da Universidade Estadual Vale do Acaraú, garantindo meios adequados de permitir máxima segurança aos participantes da pesquisa, sempre respeitando os princípios do anonimato e privacidade.

Variáveis que compuseram a análise univariada de acordo com a categoria.

\begin{tabular}{|c|c|}
\hline Condição sócio-econômica & Consumo de serviços \\
\hline Condição do domicílio & Cadastro no PSF \\
\hline Tratamento do esgoto & Recebe visita do agente comunitário de saúde \\
\hline Coleta do lixo & Freqüenta UBS \\
\hline Desnutrição & Proximidade com UBS \\
\hline Posse de plano de saúde & Onde leva a criança quando está doente \\
\hline Posse de escova & Vacinação \\
\hline Escolaridade mãe & Participação em programa de desnutrido \\
\hline Escolaridade do chefe da família & Qual programa de desnutrido \\
\hline Sexo do chefe da família & Merenda escolar \\
\hline \multicolumn{2}{|l|}{ Freqüentou creche } \\
\hline Freqüentou pré-escola & \\
\hline
\end{tabular}

PSF: Programa Saúde da Família; UBS: unidade básica de saúde. 


\section{Resultados}

Não houve recusa em participar do estudo. De acordo com a Tabela 2, uma parcela da população $(49,1 \%)$ jamais teve acesso a tratamento dentário, enquanto pouco mais da metade (50,9\%) já realizaram este tipo de tratamento em algum momento de sua vida. Pode-se observar, ainda, que $34,7 \%$, das crianças que haviam tido acesso ao tratamento dentário o realizaram há mais de um ano. A grande maioria das crianças que teve acesso a tratamento odontológico $(85,4 \%)$ o realizou na rede pública, ou seja, por intermédio do SUS. Apenas 7,5\% das crianças tiveram acesso a serviços privados, enquanto $7,1 \%$ realizaram atendimento odontológico utilizando planos de saúde ou convênios.

Tabela 2

Total e porcentagem das crianças estudadas segundo acesso ao tratamento dentário, de acordo com tipo de serviço e período da última consulta. Sobral, Ceará, Brasil, 1999-2000.

\begin{tabular}{lcc}
\hline Tratamento dentário & $\mathbf{n}$ & $\%$ \\
\hline Com acesso & 1.743 & 50,9 \\
Tipo de serviço & 972 & \\
Serviço público & 86 & 75,4 \\
Serviço privado & 80 & 7,1 \\
Convênio/Plano & & 65,3 \\
Período da consulta & 1.138 & 34,7 \\
Último ano & 605 & 49,1 \\
Mais de um & 1.682 & 100,0 \\
Sem acesso & 3.425 & \\
Total & & \\
\hline
\end{tabular}

Para identificar a relação da utilização de serviços odontológicos com as variáveis independentes do estudo, estas foram dicotomizadas em situações "adequada" e "inadequada" e avaliadas mediante análise univariada, assim como a utilização de serviços odontológicos foi definida em "sim" ou "não". Após a análise univariada, as variáveis relativas à condição sócio-econômica (característica do domicílio) e ao consumo de serviços (merenda, vacinação, cadastro no Programa Saúde da Família - PSF) não apresentaram significância estatística para explicar o uso de serviços odontológicos.

Na análise univariada, as variáveis relacionadas à condição sócio-econômica que apresentaram $\mathrm{p}<0,20$ foram: plano de saúde, posse de escova, coleta de lixo, escolaridade da mãe, tratamento do esgoto, desnutrição e escolaridade do chefe da família. Após a regressão logística, as variáveis que se apresentaram estatisticamente significativas foram: plano de saúde $(\mathrm{OR}=4,06$; IC95\%: 2,57-6,42), posse de escova $(\mathrm{OR}=3,38$; IC95\%: $2,01-5,68)$, coleta de lixo $(\mathrm{OR}=1,70$; IC95\%: 1,40-2,06), escolaridade da mãe (OR = 1,52; IC95\%: 1,29-1,79), tratamento do esgoto (OR = 1,39; IC95\%: 1,16-1,66) e desnutrição (OR = 1,22; IC95\%: 1,02-1,46).

Das variáveis relativas ao consumo de serviços, as que apresentaram $\mathrm{p}<0,20$ na relação com acesso foram: recebe visita do agente comunitário de saúde, onde leva quando doente, vacina, acesso à unidade básica de saúde, freqüência à creche e freqüência à pré-escola. Após a análise de regressão logística, a única variável que se apresentou estatisticamente significativa foi "recebe visita do agente comunitário de saúde" (OR = 0,83; IC95\%: 0,70-0,98).

O modelo final da regressão logística está bem ajustado, o que pode ser verificado pelo valor do teste de Hosmer \& Lemeshow $(0,614)$.

A distribuição dessas variáveis em relação à utilização dos serviços odontológicos, considerando-se o qui-quadrado, valor de p, a OR, o valor de $\mathrm{p}$ ajustado e a OR ajustada encontram-se na Tabela 3.

\section{Discussão}

Analisar a utilização de serviços odontológicos e os principais fatores associados à sua limitação é fundamental para podermos ter uma percepção sobre o efetivo alcance do desafio da universalização do acesso à saúde proposto pelo SUS. Apesar da importância deste tipo de estudo, existem poucas informações acerca do uso de serviços odontológicos por parte da população brasileira 12, em especial na faixa etária proposta no presente trabalho.

Entre as barreiras para acesso ao atendimento odontológico, Cohen 13 situa as relativas à sociedade (número insuficiente de ações de promoção de saúde, instalações impróprias dos serviços e reduzido apoio financeiro à pesquisa) como fundamentais a serem suplantadas, permitindo maior resposta às demandas da população.

Por meio do presente trabalho pôde-se observar a necessidade de se incrementar o atendimento público odontológico no Município de Sobral, uma vez que quase metade das crianças dos 5 aos 9 anos jamais haviam tido acesso ao cirurgião-dentista. Deve-se ressaltar que as crianças nessa faixa etária estão entre aquelas tradicionalmente priorizadas para atendimento odontológico no serviço público 14 , impondo 
Associação entre o desfecho "utilização dos serviços odontológicos" e as variáveis independentes utilizadas no estudo. Sobral, Ceará, Brasil, 1999-2000.

\begin{tabular}{|c|c|c|c|c|c|c|c|c|c|}
\hline \multirow[t]{3}{*}{ Variável } & \multicolumn{4}{|c|}{ Utilização dos serviços } & \multirow[t]{3}{*}{$\chi^{2}$} & \multirow[t]{3}{*}{$\mathbf{p}$} & \multirow[t]{3}{*}{ OR } & \multirow[t]{3}{*}{$\mathbf{P}_{\text {ajustado }}$} & \multirow[t]{3}{*}{ OR $_{\text {ajustada }}$} \\
\hline & \multicolumn{2}{|c|}{ Não } & \multicolumn{2}{|c|}{ Sim } & & & & & \\
\hline & $\mathbf{n}$ & $\%$ & $\mathrm{n}$ & $\%$ & & & & & \\
\hline \multicolumn{10}{|l|}{ Plano de saúde } \\
\hline Não & 1.640 & 50,9 & 1.581 & 41,1 & 91,40 & $<0,001$ & $5,58(3,78-8,25)$ & $<0,001$ & $4,06(2,57-6,42)$ \\
\hline Sim & 31 & 15,7 & 167 & 84,3 & & & & & \\
\hline \multicolumn{10}{|l|}{ Posse de escova } \\
\hline Não & 96 & 77,4 & 28 & 22,6 & 41,25 & $<0,001$ & $3,77(2,46-5,77)$ & $<0,001$ & $3,38(2,01-5,68)$ \\
\hline Sim & 1.571 & 47,6 & 1.727 & 52,4 & & & & & \\
\hline \multicolumn{10}{|l|}{ Lixo } \\
\hline Sem coleta & 632 & 62,2 & 384 & 37,8 & 101,7 & $<0,001$ & $2,15(1,85-2,50)$ & $<0,001$ & $1,70(1,40-2,06)$ \\
\hline Com coleta & 1.050 & 43,3 & 1.375 & 56,7 & & & & & \\
\hline \multicolumn{10}{|c|}{ Escolaridade da mãe (anos) } \\
\hline Menos de 8 & 927 & 57,1 & 697 & 42,9 & 90,36 & $<0,001$ & $1,97(1,71-2,26)$ & $<0,001$ & $1,52(1,29-1,79)$ \\
\hline Mais de 8 & 657 & 40,4 & 971 & 59,6 & & & & & \\
\hline \multicolumn{10}{|l|}{ Esgoto } \\
\hline Outro & 880 & 58,6 & 621 & 41,4 & 100,6 & $<0,001$ & $2,01(1,75-2,31)$ & $<0,001$ & $1,39(1,16-1,66)$ \\
\hline Rede pública & 800 & 41,3 & 1.036 & 58,7 & & & & & \\
\hline \multicolumn{10}{|l|}{ Desnutrição } \\
\hline Sim & 544 & 55,5 & 436 & 44,5 & 24,08 & $<0,001$ & $1,48(1,25-1,69)$ & 0,029 & $1,22(1,02-1,46)$ \\
\hline Não & 1.104 & 46,1 & 1.289 & 53,9 & & & & & \\
\hline \multicolumn{10}{|c|}{ Visita do agente comunitário de saúde } \\
\hline Às vezes/Nunca & 567 & 47,6 & 623 & 52,4 & 8,12 & 0,004 & $0,80(0,69-093)$ & 0,027 & $0,83(0,70-0,98)$ \\
\hline Sempre & 856 & 53,2 & 754 & 46,8 & & & & & \\
\hline \multicolumn{10}{|c|}{ Onde leva quando está doente } \\
\hline Outro & 262 & 37,1 & 444 & 62,9 & 48,67 & $<0,001$ & $0,55(0,46-0,65)$ & - & - \\
\hline PSF & 1412 & 51,9 & 1.307 & 48,1 & & & & & \\
\hline \multicolumn{10}{|l|}{ Vacina } \\
\hline Incompleta & 160 & 54,2 & 135 & 45,8 & 3,72 & 0,054 & $1,27(1,00-1,62)$ & - & - \\
\hline Completa & 1.480 & 48,2 & 1.592 & 51,8 & & & & & \\
\hline \multicolumn{10}{|l|}{ Acesso à UBS } \\
\hline Às vezes/Nunca & 970 & 46,5 & 1.116 & 53,5 & 12,08 & 0,001 & $0,78(0,68-0,90)$ & - & - \\
\hline Sempre & 709 & 52,6 & 638 & 47,4 & & & & & \\
\hline \multicolumn{10}{|c|}{ Escolaridade do chefe (anos) } \\
\hline Menos de 8 & 1.070 & 54,7 & 895 & 45,5 & 61,91 & $<0,001$ & $1,76(1,53-2,02)$ & - & - \\
\hline Mais de 8 & 543 & 40,5 & 799 & 59,5 & & & & & \\
\hline \multicolumn{10}{|l|}{ Freqüentou creche } \\
\hline Nunca & 1.135 & 45,4 & 1.364 & 54,6 & 8,25 & 0,004 & $0,78(0,67-0,92)$ & - & - \\
\hline Sim & 388 & 51,5 & 366 & 48,5 & & & & & \\
\hline \multicolumn{10}{|c|}{ Freqüentou a pré-escola } \\
\hline Nunca & 94 & 59,1 & 65 & 40,9 & 9,79 & 0,002 & $1,69(1,22-2,34)$ & - & - \\
\hline Freqüentou & 1.416 & 46,1 & 1.656 & 53,9 & & & & & \\
\hline
\end{tabular}

Teste Hosmer \& Lemeshow: 0,614.

PSF: Programa Saúde da Família; UBS: unidade básica de saúde.

uma discussão sobre o acesso das outras faixas etárias, em especial crianças menores de quatro anos e populações adulta e idosa. Se comparadas com os indivíduos entre 5 e 19 anos de idade, faixa etária estabelecida na PNAD/1998, 3 observase um pior desempenho na utilização de serviço odontológico em Sobral, visto que para o Brasil temos $25,2 \%$ de pessoas nesta faixa etária que nunca consultaram o cirurgião-dentista.

Pode-se observar, ainda, que das crianças que haviam tido acesso ao tratamento dentário, praticamente uma terça parte o realizaram há 
mais de um ano, o que caracteriza um acesso irregular destas crianças, possivelmente vinculado à urgência odontológica ou busca para solução de algum problema pontual. Apesar do entendimento da necessidade de incorporação de ações coletivas em saúde bucal, o acompanhamento contínuo do paciente, principalmente a partir do diagnóstico precoce das enfermidades e do tratamento imediato das enfermidades presentes, é fundamental na garantia de controle das doenças bucais e, conseqüente, melhoria na qualidade de vida. Tais resultados são próximos dos observados na PNAD/1998 3, uma vez que 57,6\% daqueles na faixa etária de 5 a 19 anos realizaram consulta odontológica durante o ano anterior à pesquisa.

Apesar da necessidade de aumento da cobertura, pode-se observar a importância do SUS na resposta ao acesso aos serviços odontológicos no município, considerando ser a grande maioria dos atendimentos realizada neste segmento. Tal situação é bastante diferente da observada por Barros \& Bertoldi 15 para a população brasileira, que teve apenas $24 \%$ (PNAD) dos atendimentos odontológicos providos pelo SUS. Sem dúvida, o objetivo do SUS é que se possa alcançar o mesmo que o observado na Suécia por Hugoson et al. 16, que sinalizam para o atendimento de todas as crianças de 3 a 15 anos pelo Serviço Público Dental.

Pelo atual estudo, o que se pôde constatar de maneira significativa foi a alta influência das condições sócio-econômicas no maior acesso aos serviços de saúde. Quando se detecta que aqueles que possuem um plano de saúde ou convênio apresentam uma possibilidade de acesso aproximadamente quatro vezes maior, mesmo em se tratando de serviços públicos, é necessária uma abordagem sobre o princípio da eqüidade. Tal situação também é observada por Pinheiro \& Torres 17 , em estudo comparativo do uso de serviços odontológicos entre os estados do Brasil. Há necessidade de uma rediscussão sobre a forma de repasse de recursos desses planos para o SUS, tendo em vista esta ser a única possibilidade de acesso a quem não tem qualquer condição de contribuir para um plano de saúde e, menos ainda, para o pagamento direto ao profissional.

Outro fator que mereceu destaque foi a posse de escova dentária, mostrando alta relação deste evento com o acesso aos serviços de saúde. Apesar da quase universalização na posse desse item (96\% das crianças), um dos elementos que pode explicar essa relação é a orientação constante dos profissionais de saúde bucal sobre a importância da higienização dentária, o que pode ter provocado um estímulo maior para as mães que desejam ver seus filhos com boas condições de saúde bucal. Por isso mesmo, seria importante que o sistema de saúde se organizasse para fornecer esse tipo de insumo ou propiciasse a sua aquisição a partir da comercialização desse item com preços mais acessíveis, na lógica da Farmácia Popular.

Ainda em relação ao componente sócio-econômico duas situações se destacaram no estudo, qual sejam, o acesso à coleta de lixo e ao tratamento de esgoto. Como é de conhecimento geral, coleta de lixo e tratamento de esgoto são benefícios diretamente relacionados com a condição de moradia, ou seja, melhor condição financeira. As crianças que apresentaram benefícios em seu domicílio apresentaram 1,7 (1,40-2,06) vez mais chance de acesso aos serviços de saúde, no caso daquelas que tinham coleta de lixo, e 1,39 (1,16$1,66)$ no caso daquelas que tinham tratamento de esgoto.

Assim como em outros estudos, a escolaridade da mãe também contribui significativamente para um maior acesso aos serviços de saúde, provavelmente pela possibilidade de maior discernimento quanto à real necessidade de tratamento da criança. Segundo Gonçalves et al. 18, a educação dá entrada a uma determinada ocupação e, portanto, a certo nível de renda, e isto pode influenciar o acesso a diferentes condutas relacionadas à saúde.

A desnutrição, uma das maiores evidências da desigualdade social, também apresentou relação com o acesso a serviço odontológico. Detectouse que as crianças desnutridas, possivelmente as mais necessitadas de cuidado, apresentaram maior dificuldade de acesso aos serviços, o que impõe a discussão de mecanismos que efetivamente permitam que os grupos mais expostos a riscos possam ter prioridade em determinadas intervenções do sistema de saúde.

Quanto ao consumo de serviços, a única variável que se apresentou estatisticamente significante foi a visita do agente comunitário de saúde. Entretanto, a não visita desse agente mostrou-se como fator de proteção, ou seja, os domicílios que não recebem sua visita apresentaram maior acesso ao tratamento odontológico. Tal situação pode estar vinculada ao fato de que os domicílios mais visitados pelos agentes comunitários de saúde serem aqueles onde as pessoas apresentam necessidade de acompanhamento mais constante de doenças sistêmicas (diabetes, tuberculose, hanseníase, problemas cardíacos) ou outras situações (desemprego, dependência de drogas, distúrbios mentais). Tais acontecimentos tornam os responsáveis incapazes de articular os cuidados a seus dependentes, entre os quais o atendimento odontológico. 
Se para Senna 19 uma primeira avaliação sobre o PSF aponta para o impacto positivo do programa em áreas com grande déficit de serviços básicos de saúde, sobretudo na Região Nordeste do país, no atual estudo fica claro que somente estratégias vinculadas ao cotidianamente trabalhado de forma tradicional pelo sistema de saúde não são suficientes. Concorda-se com Antunes et al. 20, que reforçam a necessidade de caminhos para o reconhecimento das iniqüidades no acesso à saúde bucal embasados em dados sócio-econômicos, permitindo a adoção de programas de promoção de saúde bucal compatíveis com as reais necessidades da população menos assistida.

Assim como descrito por Baldani et al. 21, entende-se que há necessidade de se implementar medidas e políticas compensatórias para amenizar os efeitos danosos das desigualdades sociais, uma vez que estas se tornam mais graves se

\section{Resumo}

O objetivo deste estudo foi analisar a utilização de serviços odontológicos pela população infantil no $\mathrm{Mu}$ nicípio de Sobral, Ceará, Brasil, relacionando-a com fatores sócio-econômicos e consumo de serviços. Foi realizado estudo transversal analítico no qual foram entrevistados 3.425 responsáveis pelas crianças para avaliar a relação entre as variáveis independentes e a utilização de serviços odontológicos. Realizou-se teste de associação pelo qui-quadrado, assim como se estimou a razão de chances e seu respectivo intervalo de confiança, utilizando-se a análise de regressão logística múltipla. Observou-se que 50,9\% das crianças tiveram acesso a serviço dentário pelo menos uma vez na vida. Desse total, 65,3\% o realizaram no decorrer do último ano pesquisado, sendo que $85,4 \%$ na rede pública. Pode-se observar a importância do Sistema Único de Saúde na resposta ao acesso aos serviços odontológicos, considerando ser a grande maioria dos atendimentos realizada neste segmento. Observou-se, ainda, que os fatores que mais afetaram a utilização do serviço odontológico foram os vinculados à condição sócio-econômica, destacando-se o fato de ter plano de saúde, posse de escova dentária, coleta de lixo, escolaridade da mãe, tratamento do esgoto e desnutrição.

Acesso aos Serviços de Saúde; Assistência Odontológica para Crianças; Saúde Bucal estratégias não são capazes de prover ao menos benefícios equivalentes para grupos de baixo nível sócio-econômico.

O desafio de formuladores e gestores de políticas públicas no Brasil é, segundo Narvai et al. 22 , o de propor medidas sociais e econômicas mais gerais, voltadas ao enfrentamento da exclusão social e intervenções de saúde pública complementares, dirigidas aos grupos mais vulneráveis, tanto no campo da promoção da saúde bucal quanto no âmbito mais restrito da assistência odontológica.

É fundamental que o princípio da eqüidade, entendido enquanto a perspectiva de ampliação de acesso a setores sociais tradicionalmente excluídos, seja efetivamente discutido pela sociedade, profissionais de saúde e gestores, visando a eliminar as iniqüidades e proporcionar maior justiça social.

\section{Colaboradores}

L. R. A. Noro e A. G. Roncalli realizaram a revisão sistemática da literatura e participaram de todas as etapas de elaboração do artigo. F. I. R. Mendes Júnior participou da elaboração do instrumento, acompanhou e coordenou a coleta de dados e colaborou na redação final do artigo. K. C. Lima colaborou na revisão sistemática da literatura, participou da discussão dos resultados e da redação final do artigo. 


\section{Referências}

1. Brasil. Lei $\mathrm{n}^{\circ}$. 8.080, de 19 de setembro de 1990 . Dispõe sobre as condições para a promoção, proteção e recuperação da saúde, a organização e o funcionamento dos serviços correspondentes e dá outras providências. Diário Oficial da República Federativa do Brasil 1990; 20 set.

2. Gomes PR, Costa SC, Cypriano S, Sousa MLR. Paulínia, São Paulo, Brasil: situação da cárie dentária em relação às metas OMS 2000 e 2010. Cad Saúde Pública 2004; 20:866-70.

3. Instituto Brasileiro de Geografia e Estatística. Pesquisa nacional por amostra de domicílios, 1998. http://www.ibge.gov.br/estatistica/populacao/ trabalhoerendimento/pnad98/saude/sb11.shtm (acessado em 18/Set/2007).

4. Schwarz E. Access to oral health care - an Australian perspective. Community Dent Oral Epidemiol 2006; 34:225-31.

5. Ferreira AAA, Piuvezam G, Werner CWA, Alves MSCF. A dor e a perda dentária: representações sociais do cuidado à saúde bucal. Ciênc Saúde Coletiva 2006; 11:211-8.

6. Manfredini M. Abrindo a boca: reflexões sobre bocas, corações e mentes. In: Campos FCB, Henriques CMP, organizadores. Contra a maré à beiramar. São Paulo: Editora Hucitec; 1997. p. 78-87.

7. Sanders AE, Slade GD. Deficits in perceptions of oral heath to general health in populations. J Public Health Dent 2006; 66:255-62.

8. Fernandes LS, Peres MA. Associação entre atenção básica em saúde bucal e indicadores socioeconômicos municipais. Rev Saúde Pública 2005; 39:930-6.

9. Ministério da Saúde. Diretrizes da política nacional de saúde bucal. http://www.saude.gov.br/ bucal (acessado em 10/Set/2007).

10. Instituto Brasileiro de Geografia e Estatística. Censo demográfico. Brasília: Instituto Brasileiro de Geografia e Estatística; 1996.

11. Barreto ICHC. Morbidade referida e seus condicionantes em crianças de 5 a 9 anos da zona urbana de Sobral-CE [Tese de Doutorado]. São Paulo: Faculdade de Medicina, Universidade de São Paulo; 2006.
12. Albuquerque OMR, Abegg C, Rodrigues CS. Percepção de gestantes do Programa Saúde da Família em relação às barreiras no atendimento odontológico em Pernambuco, Brasil. Cad Saúde Pública 2004; 20:789-96.

13. Cohen LK. Converting unmet need for care to effective demand. Int Dent J 1987; 37:114-6.

14. Lacerda JT, Simionato EM, Peres KG, Peres MA, Traebert J, Marcenes W. Dor de origem dental como motivo de consulta odontológica em uma população adulta. Rev Saúde Pública 2004; 38:453-8.

15. Barros AJD, Bertoldi AD. Desigualdades na utilização e no acesso a serviços odontológicos: uma avaliação em nível nacional. Ciênc Saúde Coletiva 2002; 7:709-17.

16. Hugoson A, Koch G, Göthberg C, Helkimo AN, Lundin SA, Norderyd O, et al. Oral health of individuals aged 3-80 years in Jönköping, Sweden during 30 years (1973-2003). I. Review of findings on dental care habits and knowledge of oral health. Swed Dent J 2005; 29:125-38.

17. Pinheiro RS, Torres TZG. Uso de serviços odontológicos entre os Estados do Brasil. Ciênc Saúde Coletiva 2006; 11:999-1010.

18. Gonçalves ER, Peres MA, Marcenes W. Cárie dentária e condições sócio-econômicas: um estudo transversal com jovens de 18 anos de Florianópolis, Santa Catarina, Brasil. Cad Saúde Pública 2002; 18:699-706.

19. Senna MCM. Eqüidade e política de saúde: algumas reflexões sobre o Programa Saúde da Família. Cad Saúde Pública 2002; 18:203-11.

20. Antunes JL, Jahn GM, Camargo MA. Increasing inequalities in the distribution of dental caries in the Brazilian context in Finland. Community Dent Health 2005; 22:94-100.

21. Baldani MH, Vasconcelos AGG, Antunes JLF. Associação do índice CPO-D com indicadores sócioeconômicos e de provisão de serviços odontológicos no Estado do Paraná, Brasil. Cad Saúde Pública 2004; 20:143-52.

22. Narvai P, Frazão P, Roncalli A, Antunes J. Cárie dentária no Brasil: declínio, polarização, iniqüidade e exclusão social. Rev Panam Salud Pública 2006; 19:385-93.

Recebido em 06/Jul/2007

Versão final reapresentada em 08/Nov/2007 Aprovado em 04/Dez/2007 